\title{
Time-Lapse 3-D Seismic Wave Simulation via the Generalized Multiscale Finite Element Method
}

\author{
Yongchae Cho ${ }^{1, *}$, Richard L. Gibson, Jr. ${ }^{2}$, Hyunmin $\mathrm{Kim}^{3}$, \\ Mikhail Artemyev ${ }^{4}$ and Yalchin Efendiev ${ }^{5,6}$ \\ 1 Shell International Exploration E Production Inc., 150 N Dairy Ashford Road, \\ Houston, TX 77079, USA. \\ 2 Department of Geology E Geophysics, Texas AEM University, College Station, \\ Texas 77843-3115, USA. \\ 3 Shell Exploration \& Production Company, 701 Poydras Street, New Orleans, \\ LA 70139, USA. \\ ${ }^{4}$ Arista Networks, Dublin, Ireland. \\ ${ }^{5}$ Department of Mathematics, Texas AEM University, College Station, \\ Texas 77843-3368, USA. \\ ${ }^{6}$ Multiscale Model Reduction Laboratory, North-Eastern Federal University, Yakutsk, \\ Republic of Sakha 677980, Russia .
}

Received 1 April 2018; Accepted (in revised version) 28 October 2018

\begin{abstract}
Numerical solution of time-lapse seismic monitoring problems can be challenging due to the presence of finely layered reservoirs. Repetitive wave modeling using fine layered meshes also adds more computational cost. Conventional approaches such as finite difference and finite element methods may be prohibitively expensive if the whole domain is discretized with the cells corresponding to the grid in the reservoir subdomain. A common approach in this case is to use homogenization techniques to upscale properties of subsurface media and assign the background properties to coarser grid; however, inappropriate application of upscaling might result in a distortion of the model, which hinders accurate monitoring of the fluid change in subsurface. In this work, we instead investigate capabilities of a multiscale method that can deal with fine scale heterogeneities of the reservoir layer and more coarsely meshed rock properties in the surrounding domains in the same fashion. To address the 3-D wave problems, we also demonstrate how the multiscale wave modeling technique can detect the changes caused by fluid movement while the hydrocarbon production activity proceeds.
\end{abstract}

AMS subject classifications: 86A15

Key words: Fluid simulation, time lapse, Generalized multiscale finite element, elastic wave.

*Corresponding author. Email addresses: ycho.geo@gmail . com (Y. Cho), gibson@tamu .edu (R. L. Gibson, Jr.), hmkim1030@tamu.edu (H. Kim), artemiev.mikhail@gmail.com (M. Artemyev), yalchinrefendiev@gmail.com (Y. Efendiev) 


\section{Introduction}

Time-lapse seismic monitoring problem requires a large amount of computational resources since it needs repetitive seismic imaging and waveform inversion. A classical approach of $4 \mathrm{D}$ seismic data processing is often done by migrating baseline and monitoring data on the same smooth tomographic model. In the $4 \mathrm{D}$ seismic images, we can observe that the images are misaligned due to the change of the fluid composition in the reservoir by calculating time-shifts or applying post-stack alignment by the computation of image difference products. We can apply ray theory based approach such as Kirchhoff migration for the reservoir with relatively simple structures. However, when the reservoir includes a complicated structure (i.e., salt diapir or karsts), we often take a waveequation based method to resolve the seismic images with complex geology. In this case, an accurate simulation of seismic wave propagation is important, and the computational burden becomes more critical when we perform the wave modeling in 3D models.

In addition, characterization of the physical properties of a reservoir for flow simulations is typically performed on a coarser scale than a general seismic resolution, and that creates significant challenges for classical methods for seismic wave simulation such as finite difference or continuous-Galerkin finite element methods, because in many cases the domain is discretized according to the smallest elements. When the size of the grid cell used in flow simulations is greater than the scale of earth model (or seismic), we often need to apply an upscaling or averaging technique. This might incur a distortion of the earth properties, and too large size of the grid cell hinders a stable simulation of seismic waves with high frequency. In spite of expensive computational cost, to capture the details of the change in reservoir we need accurate simulation of seismic waves and this calculation should be repeated at time intervals corresponding to monitoring surveys. Therefore, many studies have been done to develop full wavefield modeling methods and have demonstrated a method to optimize them for time-lapse calculations. For example, Malcolm and Willemsen [1] proposed an optimization technique where a local solver is used around the time-lapse region to prevent recomputation of the wavefield in the unchanged overburden.

A popular approach for solving the wave equation numerically is to apply the finite difference method (FDM) [2-7]. FDM is widely used due to its easiness for discretization; however, it also has inherent drawbacks such as the problem of free surface topography and less flexibility to deal with an unstructured mesh. The finite element method (FEM), in contrast, provides the solutions for more flexible mesh structuring, which can model the complex topography or fracture networks. Various FEM approach have been proposed, and one classical method is continuous-Galerkin (CG) FEM [8-10]. However, compared to the other FEM methods, CG FEM might be computationally expensive since the global matrix is not diagonal or block diagonal, which requires nontrivial sparse-matrix operations for multiplication of mass and stiffness matrices with the wave solutions at each time step. As a solution of this issue, spectral element method (SEM) is proposed by Komatitsch et al. [11-13]. Nevertheless, CG FEM still has limita- 
tion in that all the solutions are continuous on the boundary of the elements. Thus, the wave solution can be inaccurate when we handle a media with high impedance contrast, especially around the high contrast layer interface. This problem is removed when we consider discontinuous-Galerkin (DG) FEM, which is initially developed for the elliptic problem [14-16] and transport equation [17]. The DG FEM has been more widely used, and also applied for the time-dependent wave problems [18-21]. Nevertheless, DG FEM has its own weakness such as more complicated implementation and dispersion analysis; however, it has strong advantage over CG FEM in that the global mass matrix is block diagonal, which makes it easier to solve the system in wave problem with large 3-D model.

Regardless of the numerical approach for discretizing the wave equation, modeling 3-D seismic waves for the time-lapse problem becomes a computationally intensive task. Most of the codes for seismic wave propagation are designed to be scalable up to hundreds and even thousands of computing cores (i.e., SPECFEM3D implementing Spectral Element Method [12], or SOFI3D implementing Finite Differences, [22]). Some of the realizations are especially attractive for usage on modern supercomputers as they take advantages of powerful and energy efficient Graphics Processing Units (GPUs) [23]. Such implementations are still rare, however, because efficient use of GPUs requires nontrivial programming and redevelopment of classical algorithms unless they were suitable for GPU programming model from the beginning.

Recent development of a new class of methods, commonly referred as "multiscale methods, allowed solution of these problems in a more efficient way. Compared to the conventional implementation of FEM, a remarkable feature of multiscale FEM (MsFEM) [24-26] is to have the basis functions incorporate the fine scale heterogeneity of the background property model, while the classical FEM uses identical basis function, which is independent to the model properties throughout the entire model domain. Chung et al. $[27,28]$ and Gibson et al. [29] applied the concept of MsFEM to the wave problem. Then, Efendiev et al. $[30,31]$ proposed to utilize multiple multiscale basis functions that are computed from the appropriately defined local spectral problem, which method is called as the generalized multiscale FEM (GMsFEM). Chung et al. [32] introduced the GMsFEM using DG method for solving the second-order wave equation, and they proposed the method to apply two different types of basis functions (i.e., interior and boundary basis) to capture the fine scale heterogeneity of the media on coarse scale without performing any upscaling method. Cho et al. [33] applied the GMsFEM to simulate the elastic waves in the fracture media to efficiently incorporate the fine-scale fractures without performing a model upscaling. The advantages of the GMsFEM can be defined from two points of view. First, the multiscale methods reduce the computational cost of the simulations by solving problems on a coarse scale by means of special multiscale basis functions that take the fine scale heterogeneities into account and maintain the accuracy of the approximation. Secondly, most of the multiscale approaches are embarrassingly parallel, which means that scalable algorithms exist, and they can be efficiently implemented for modern distributed supercomputer architectures. 
The main goal of this research is to apply the DG based GMsFEM approach to timelapse wave simulation combined with the three-phase (gas, oil, and water) fluid simulation. In this paper, we investigate how the GMsFEM, which has been successfully used for many applications including seismic wave simulations [27,32,34], can be used for the time-lapse problems and how this method can be implemented keeping modern supercomputer architecture in mind to approach 3-D problems.

\section{Theory \& method}

In this paper, we demonstrate that we can effectively monitor the changes in seismic due to reservoir changes. Also, we show that the capability of the GMsFEM wave modeling engine is useful for time-lapse seismic monitoring as the hydrocarbon production proceeds. We first introduce the physics that is used for the fluid simulation. Next, we illustrate the method which is employed for building background properties (i.e., density, $\mathrm{P}$ - and S-wave velocity) that are required for the wave simulation. We then describe the detailed implementation of the GMsFEM in the last part of this section.

\subsection{Fluid flow simulation}

For simulating the fluid behavior over the production period, we used Schlumberger Eclipse software for black oil simulators, which considers three different phases of fluids: water, oil, and gas. In black oil equation [35], water flow is simulated explicitly together with other hydrocarbon components. The black oil equation can be expressed as

$$
\begin{aligned}
& \frac{\partial}{\partial t}\left(\phi \frac{S_{w}}{B_{w}}\right)+\nabla \cdot\left(\frac{1}{B_{o}} \vec{u}_{w}\right)=0 \\
& \frac{\partial}{\partial t}\left[\phi\left(\frac{S_{o}}{B_{o}}+\frac{R_{V} S_{g}}{B_{g}}\right)\right]+\nabla \cdot\left(\frac{1}{B_{o}} \vec{u}_{o}+\frac{R_{V}}{B_{g}} \vec{u}_{g}\right)=0, \\
& \frac{\partial}{\partial t}\left[\phi\left(\frac{R_{S} S_{o}}{B_{o}}+\frac{S_{g}}{B_{g}}\right)\right]+\nabla \cdot\left(\frac{R_{S}}{B_{o}} \vec{u}_{o}+\frac{1}{B_{g}} \vec{u}_{g}\right)=0
\end{aligned}
$$

where $B$ and $S$ denote volume factor and fluid saturation, respectively, and the corresponding phases are noted by the subscript: $o$ oil, $g$ gas, and $w$ water. $\vec{u}_{w}, \vec{u}_{o}, \vec{u}_{g}$ are Darcy velocities of the water, oil, and gas phase in reservoirs. $R_{V}$ is a vaporized oil in gas phase. $R_{S}$ is the ratio of volume of gas to the volume of oil at standard conditions, which represents the phase change between the oil and gas. In detail, $R_{S}$ becomes smaller as the pressure decreases, and the amount of gas dissolved in oil is reduced as the magnitude of $R_{S}$ decreases. Therefore, as the pressure reduces, the volume of free gas in the reservoir increases.

In this paper, we assumed the gas takes the largest portion in the pore volume. Also, by applying the black-oil relationship (Eq. (2.1)), we took the phase change of the gas 
and oil into consideration. This is important, especially when we deal with the flow simulation including gas, since large quantities of gas can come out of the solution if parts of the reservoir gradually drops below bubble point during production.

For executing the black oil simulator, porosity $\phi$ and permeability $K$ volumes are the main inputs, and desired outputs would be the volumes of fluid with corresponding effective pressure. In this study, we assume that the temperature is constant. By using the information of the fluid saturation, we will extract the velocity and density information as presented in the following subsection.

\subsection{Seismic property model construction}

We applied Eberhart-Phillips's method [36] to set the initial velocity by using the fluid simulation results. In their work, a multivariate analysis is performed to define an empirical relationships among seismic velocity and relative rock properties: effective pressure, porosity, and clay content in sandstone. According to the empirical relationship [36], Pand $S$-wave velocities can be defined as a function of porosity $\phi$, clay content of sandstone $C$, and effective pressure $P_{e}$ as follows:

$$
\begin{gathered}
V_{p}^{*}=5.77-6.94 \phi-1.73 \sqrt{C}+0.446\left(P_{e}-e^{-16.7 P_{e}}\right), \\
V_{s}=3.70-4.94 \phi-1.57 \sqrt{C}+0.361\left(P_{e}-e^{-16.7 P_{e}}\right) .
\end{gathered}
$$

We then additionally considered the velocity of the fluid since the information of the fluid composition is missing in above relation. Given that $V_{s}$ is less sensitive to the fluid components, we applied a correction to $V_{p}$ relation as

$$
\frac{1}{V_{p}}=\frac{1-\phi}{V_{p}^{*}}+\frac{\phi_{g}}{V_{g}}+\frac{\phi_{w}}{V_{w}}+\frac{\phi_{o}}{V_{o}}
$$

where subscript $g, w$, and $o$ denote gas, water, and oil, respectively. We suppose that the whole pore volume is filled with the fluids $\left(\phi=\phi_{g}+\phi_{w}+\phi_{0}\right)$. We also assume that the velocity of the fluid as follows: $V_{g}=0.480 \mathrm{~km} / \mathrm{s}, V_{w}=1.5 \mathrm{~km} / \mathrm{s}$, and $V_{o}=1.3 \mathrm{~km} / \mathrm{s}$. We calculated the density volume by taking average of the rock and fluid velocity based on the ratio as

$$
\rho=(1-\phi) \rho_{g r}+\phi_{g} \rho_{g}+\phi_{w} \rho_{w}+\phi_{o} \rho_{o},
$$

where $\rho$ means the bulk density, and we assume that the whole rock consists of sandstone $\left(\rho_{g r} \approx 2.67 \mathrm{~g} / \mathrm{cm}^{3}\right)$. We are to use the background properties $\left(V_{p}, V_{s}, \rho\right)$ that are derived from above relations for the multiscale wave modeling.

\subsection{Seismic simulation via generalized multiscale finite element method}

To solve the isotropic elastic wave equation, we followed the formulation which is described in Gao et al. [37]. Here we give a brief overview of ideas behind it, and its 
main formulations to implement the GMsFEM. We solve the elastic wave equation in the bounded 3-D domain $\Omega \subset \mathbb{R}^{3}$

$$
\begin{aligned}
& \rho \partial_{t}^{2} \mathbf{u}-\nabla \cdot \boldsymbol{\sigma}=\mathbf{f}, \quad(\boldsymbol{\sigma}=\mathbf{c}: \varepsilon), \\
& \varepsilon=\frac{1}{2}\left[\nabla \mathbf{u}+(\nabla \mathbf{u})^{\mathrm{T}}\right],
\end{aligned}
$$

where $\mathbf{u}(\mathbf{x}, t)$ displacement, $\rho(x)$ density of the medium, $\sigma(\mathbf{u})$ stress tensor, $\varepsilon(\mathbf{u})$ strain tensor, $\mathbf{c}(\mathbf{x})$ fourth-rank elasticity tensor, and $\mathbf{f}(\mathbf{x}, t)$ external source term. We apply the absorbing boundary condition which is implemented as absorbing layers by increasing damping (ALID) [38] on all surfaces of the domain. Source function located at $\mathbf{x}_{0}$ is represented as a product of a spatial force $G(\mathbf{x})$ and the Ricker wavelet in time $\mathbf{f}=G(\mathbf{x}) \mathbf{P}(\theta) R(t)$, where $\mathbf{P}(\theta)$ controls the direction of the source as follows: $\mathbf{P}(\theta)=$ $(\cos \theta, \sin \theta)^{\mathrm{T}}$. The Gaussian-correlated source term $G$ and the time dependent source function $R(t)$ can be expressed as

$$
\begin{aligned}
& G(\mathbf{x})=\exp \left[-\left(\frac{\mathbf{x}-\mathbf{x}_{0}}{7 h}\right)^{2}\right], \\
& R(t)=\left(1-2 \psi^{2}\right) \exp \left(-\psi^{2}\right),
\end{aligned}
$$

where $\mathbf{x}_{0}$ is the source position, and $h$ is the size of the fine scale element in fine mesh. $\psi=\pi f_{0}\left(t-t_{0}\right)$ includes the central frequency of the wavelet $f_{0}$ with corresponding time delay $t-t_{0}$.

GMsFEM operates on two grids (Fig. 1) with different cell size: a coarse mesh $\mathscr{T}^{H}$ and a fine mesh $\mathscr{T}^{h}$, where $h \ll H$. The algorithm of this multiscale method is typically split in two stages for convenience of understanding and implementation: the offline and online stages. In the offline stage, we construct the basis functions on a coarse cell, which incorporate the fine scale heterogeneity of the background properties (i.e., velocity and density). Here, the key goal of the GMsFEM is to incorporate the highly heterogenous background properties into the wave modeling without applying any upscaling technique. In the online stage, we perform the actual wave simulation in coarse grid by using the basis functions that are built in the previous offline stage.

\subsubsection{Offline stage: computation of basis functions}

The offline stage includes preliminary computations and does not involve the solution of the underlying wave propagation problem. The idea of coarsening in the GMsFEM is in using the so-called multiscale basis functions that are computed on the fine grid $\mathscr{T}^{h}$ within each coarse element $K \in \mathscr{T}^{H}$. These basis functions, therefore, take into account the fine scale heterogeneities of media such as finely layered reservoir domains. To construct the basis functions in Discontinuous-Galerkin (DG) formulation, we split the space spanned by the multiscale basis functions in two subspaces $V_{H}(K)=V_{H}^{1}(K) \oplus V_{H}^{2}(K)$. The subspace $V_{H}^{2}$ is defined to compute the eigenmodes of the interior part the coarse element 


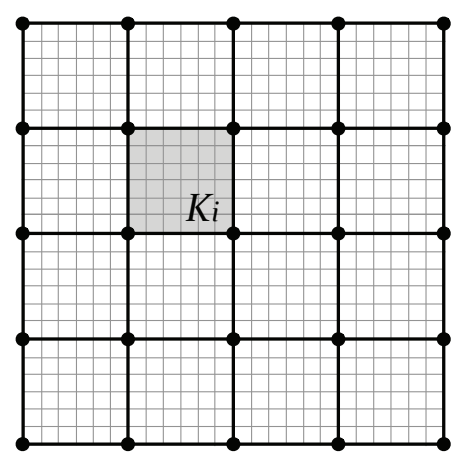

Figure 1: Schematic sketch of the multiscale mesh with two different sizes: fine and coarse grids.

$K$, while another subspace $V_{H}^{1}$ considers the local eigenvalue problem at the boundary of each coarse element $\partial K$.

To build the subspace $V_{H}^{1}(K)$ of boundary basis functions, we start with the local linear elasticity problem which can be written as

$$
\begin{aligned}
& -\nabla \cdot(\mathbf{c}: \varepsilon)=0 \quad \text { in } K, \\
& \boldsymbol{\varepsilon}=\frac{1}{2}\left[\nabla \mathbf{w}+(\nabla \mathbf{w})^{\mathrm{T}}\right], \\
& \mathbf{w}=\delta_{j} \quad \text { on } \partial K,
\end{aligned}
$$

where $\delta$ is the delta function, and $j$ indexes boundary nodes on $\partial K$ including fine nodes from $h$ around the shaded cell in Fig. 1 . Then the snapshot space $\tilde{W}_{H}^{1}[31,39]$ (a linear span of the harmonic extensions shown in Eq. (2.7)) can be defined as

$$
\tilde{W}_{H}^{1}=\operatorname{span}\left\{\mathbf{w}_{1}, \mathbf{w}_{2}, \cdots, \mathbf{w}_{d p}\right\},
$$

where $d$ is the level of spatial dimension ( 3 in this case), and $p$ is the total number of finescale boundary nodes of a coarse cell. When we deal with large model in 3-D problems, the test function space includes a significant number of eigenvectors and corresponding eigenfunctions. Hence, for practical implementation, we select only a few important modes from the snapshot space $\tilde{W}_{H}^{1}$ to the actual space of the boundary basis functions $V_{H}^{1}$. The important modes are obtained by solving the following eigenproblem:

$$
\left\{\int_{K} \sigma\left(\mathbf{w}_{\mu}\right): \varepsilon(\mathbf{v}) d \mathbf{x}\right\} \hat{\mathbf{u}}=\mu\left\{\int_{\partial K} \rho \mathbf{w}_{\mu} \cdot \mathbf{v} d S\right\} \hat{\mathbf{u}}, \quad \forall \mathbf{v} \in W_{H}(K),
$$

with $\mathbf{w}, \mathbf{v} \in \tilde{W}_{H}^{1}$. However, by considering the first $N_{b}$ eigenvectors $\mathbf{q}=\left\{\hat{u}_{1}, \hat{u}_{2}, \cdots, \hat{u}_{N_{b}}\right\}$ corresponding to the smallest eigenvalues $\mu_{1} \leq \cdots \leq \mu_{N_{b}} \leq \cdots \leq \mu_{\text {all }}$, we can take the most dominant wave modes into consideration for the wave modeling. Given that the form of the generalized eigenvalue problem is similar to the frequency-domain wave equation, the solution of the local eigenvalue problem can be analogous to the dominant wave frequencies. To put it differently, lower wave modes (or smaller $\mu$ ) include longer wavelength 
information, while higher modes cover the wave components from shorter wavelength. In this regard, the final subspace $V_{H}^{1}$ can be expressed as $V_{H}^{1}=\operatorname{span}\left\{\varphi_{1}, \varphi_{2}, \cdots, \varphi_{N_{b}}\right\}$. The subspace $V_{H}^{1}(K)$ is therefore spanned by the basis functions as

$$
\varphi_{i, k}=\Sigma \mathbf{q}_{i, j} \mathbf{w}_{j, k}, \quad\left(i=1,2, \cdots, N_{b}\right),
$$

where the indexes $i$ and $j$ denote the $j$-th node in the $i$-the vector.

To build the subspace $V_{H}^{2}(K)$ of interior basis functions, we need to find the pair

$$
\begin{aligned}
& -\nabla \cdot(\mathbf{c}: \varepsilon)=\lambda \rho \mathbf{w} \text { in } K, \\
& \varepsilon=\frac{1}{2}\left[\nabla \mathbf{w}+(\nabla \mathbf{w})^{\mathrm{T}}\right],
\end{aligned}
$$

where we set zero Dirichlet boundary condition as $\mathbf{w}=0$. Then local problem shown in (2.11) corresponds with the following system:

$$
\left\{\int_{K_{\mathrm{in}}} \sigma(\mathbf{u}): \varepsilon(\mathbf{v}) d \mathbf{x}\right\} \mathbf{z}_{\lambda}=\lambda\left\{\int_{K_{\mathrm{in}}} \rho \mathbf{w} \cdot \mathbf{v} d \mathbf{x}\right\} \mathbf{z}_{\lambda}, \quad \forall \mathbf{v} \in V_{h}^{0}(K)
$$

where $V_{h}^{0}(K)$ is a space of fine scale bilinear basis functions that are zero on $\partial K . K_{\text {in }}$ means the nodes that are not on $\partial K$, but in inner part of the coarse element $K$. Similarly, as shown in the space of the boundary basis functions, we choose the first $N_{i}$ eigenvectors corresponding to the smallest eigenvalues $\lambda_{1} \leq \cdots \leq \lambda_{N_{i}} \leq \cdots \leq \lambda_{\text {all }}$, serving as the interior bases $V_{H}^{2}(K)=\operatorname{span}\left\{\phi_{1}, \phi_{2}, \cdots, \phi_{N_{i}}\right\}$. Thus, the final multiscale basis function space $V_{H}(K)=V_{H}^{1}(K) \oplus V_{H}^{2}(K)$, which consists of the subspace of the interior basis and the boundary basis, can be written as

$$
\begin{aligned}
& V_{H}^{1}=\operatorname{span}\left\{\varphi_{1}, \varphi_{2}, \cdots, \varphi_{N_{b}}\right\}, \\
& V_{H}^{2}=\operatorname{span}\left\{\phi_{1}, \phi_{2}, \cdots, \phi_{N_{i}}\right\} .
\end{aligned}
$$

In the GMsFEM, these basis functions are computed only once for a specific model, and all simulations are computed on the coarse grid. In other words, the multiscale basis functions are independent of the source and receiver locations. Thus, this advantage becomes greater when we simulate repetitive multiple shots and receiver sets such as the wave equation based imaging or full-waveform inversion cases. Especially, in full-waveform inversion with the GMsFEM wave modeling engine, the seismic property model is updated in each iteration, so we would have to go back to the offline stage to alter the basis functions. However, as we can greatly accelerate the wave modeling while we loop over multiple shots, repetitive offline stage adds little effort to the total computation. Also, these basis functions incorporate the most dominant modes computed from the local spectral problems, so utilizing them for the wave simulation on coarse grid can greatly accelerate computations.

For the actual implementation of the offline stage, we applied the oversampling technique to enhance the accuracy of the GMsFEM solutions. The main goal of oversampling 
is to reduce the influence of the fixed boundary conditions that are prescribed on a certain coarse element $K$ when we solve the local spectral problems. The concept of the oversampling technique is that we consider a larger domain of the coarse element $K_{o s}$ than the actual size of coarse element $K$ as presented in Fig. 1 as a gray area. However, we still maintain the boundary conditions and the formulations of the local eigenvalue problem. After obtaining the solutions of the eigenproblem on the extended region $K_{o s}$, we resample the value on the inner part, which corresponds to the original region of $K$. Through this oversampling technique, we can reduce the contribution of the prescribed boundary condition of the local problem so the final multiscale basis functions have a better representation of the fine-scale heterogeneity of the background properties. Gao et al. [37] demonstrated that the oversampling plays an important role in reducing the error of the wave solutions from the GMsFEM, and this becomes more critical when we deal with a discontinuous-Galerkin formulation than a continuous-Galerkin approach.

\subsubsection{Online stage: wave simulation in coarse mesh}

At the online stage we actually solve the underlying wave equation in a significantly reduced coarse scale space $V_{H}$. The first task is to compose the global mass and stiffness matrices by projecting the fine scale matrices to the coarse scale. We assemble the fine scale global matrices $\mathbf{M}_{h}, \mathbf{K}_{h}$, and the source vector $\mathbf{F}_{h}$ by following the conventional finite element assembly approach. We then build a global projection matrix $\mathbf{R}$, which consists of the multiscale basis functions as

$$
\mathbf{R}=\left(R_{1}, R_{2}, R_{3}, \cdots, R_{N}\right)^{\mathrm{T}},
$$

where $N$ is the total number of coarse elements, and the component of each row of the global projection matrix can be expressed as

$$
R_{i}=\left[\Phi_{i, 1}, \Phi_{i, 2}, \cdots, \Phi_{i, j}\right],
$$

with $\Phi_{i, j}$ being the $j$-th multiscale basis function at $i$-th coarse node, which is determined by Eq. (2.13). Thus, the dimension of the global projection matrix $\mathbf{R}$ would be $\sum_{j=1}^{N}\left(N_{b}+\right.$ $\left.N_{i}\right)_{j} \times n$, where $n$ is the number of degrees of freedom of the mesh $\mathscr{T}^{h}$. By applying the global projection matrix, we can compute the global mass and stiffness matrices in coarse mesh as follows:

$$
\mathbf{M}_{H}=\mathbf{R M}_{h} \mathbf{R}^{\mathrm{T}}, \quad \mathbf{K}_{H}=\mathbf{R} \mathbf{K}_{h} \mathbf{R}^{\mathrm{T}}, \quad \mathbf{F}_{H}=\mathbf{R F}_{h} .
$$

By using the coarse scale matrices, the wave simulation can be performed in the coarse mesh. We employed a second order explicit time scheme for solving the wave equation

$$
\mathbf{M}_{H} \frac{\mathbf{U}_{H}^{n+1}-2 \mathbf{U}_{H}^{n}+\mathbf{U}_{H}^{n-1}}{\Delta t^{2}}+\mathbf{K}_{H} \mathbf{U}_{H}^{n}=\mathbf{F}_{H}^{n}+\mathbf{D}_{H} \frac{\mathbf{U}_{H}^{n+1}-\mathbf{U}_{H}^{n-1}}{2 \Delta t},
$$

where $\mathbf{M}_{H}, \mathbf{K}_{H}$ and $\mathbf{D}_{H}$ are mass, stiffness and damping (for absorbing boundary condition) coarse scale matrices, respectively. The GMsFEM uses the Symmetric Interior 
Penalty Discontinuous-Galerkin (SIPDG) method [37,40,41] for assembling these coarse scale matrices. Solving Eq. (2.17) yields the solution in coarse mesh. Regarding the stability condition of the GMsFEM, we can apply the similar range of $\Delta t$ to the time marching as defined in the fine-scale solution. A rigorous demonstration of the stability and dispersion relation of the GMsFEM in solving elastic wave equation might be beyond the scope of the paper. However, relative demonstrations can be found from other researchers' work. For example, Chung et al. [42] presented a rigorous proof of the stability and convergence of the similar multiscale method for solving the acoustic wave equation. Gibson et al. [29] also demonstrated some examples of the numerical analysis of dispersion error for different GMsFEM parameters in the acoustic wave problem. In addition, Gao et al. [37] showed multiple numerical examples of stability condition for conventional CG and DG FEM in the multiscale method. Considering a rough estimation of the accuracy, the number of basis functions around $10 \%$ of the degree of freedom provides admissible range of errors. After obtaining the coarse scale solutions, we can reproject the coarse scale solutions into fine mesh as

$$
\mathbf{U}_{h}=\mathbf{R}^{\mathrm{T}} \mathbf{U}_{H},
$$

where $\mathbf{U}_{h}$ has the same dimension with the fine scale grid.

\subsection{Parallel implementation}

Each of the two stages of the computational procedure of the method can benefit from different types of parallelism. At the offline stage the computation of multiscale basis functions can be performed independently for each coarse element $K \in \mathscr{T}^{H}$. Therefore, the offline algorithm belongs to the class of embarrassingly parallel ones, and should be essentially implemented with the MPI paradigm. Our experiments confirm a linear speedup when the number of computing cores increases up to the number of coarse cells.

At the online stage, when the solution of the wave equation is performed on a coarse scale, the most computationally intensive part is the matrix-vector multiplication for the projection of fine-scale mass and stiffness matrices $\left(\mathbf{M}_{h}\right.$ and $\left.\mathbf{K}_{h}\right)$ into coarse-scale matrices $\left(\mathbf{M}_{H}\right.$ and $\left.\mathbf{M}_{H}\right)$ by applying Eq. (2.16), and therefore it can also run in parallel. After implementing this stage with OpenMP and CUDA parallel paradigms (using cuSparse library), we conclude that the performance of the latter is much higher, and therefore desirable for the parallel execution of much more computationally intensive 3-D problems.

\section{Synthetic reservoir model}

We selected the SPE-10 [43] model (Fig. 2) for testing the multiscale time-lapse wave simulation. The original dimension of the model is $60 \times 220 \times 85$; however, in this case, we only considers a part of the model $60 \times 60 \times 80$ to have a better observation on the seismic waves. The model consists of two main zones - upper and lower zone represent the 


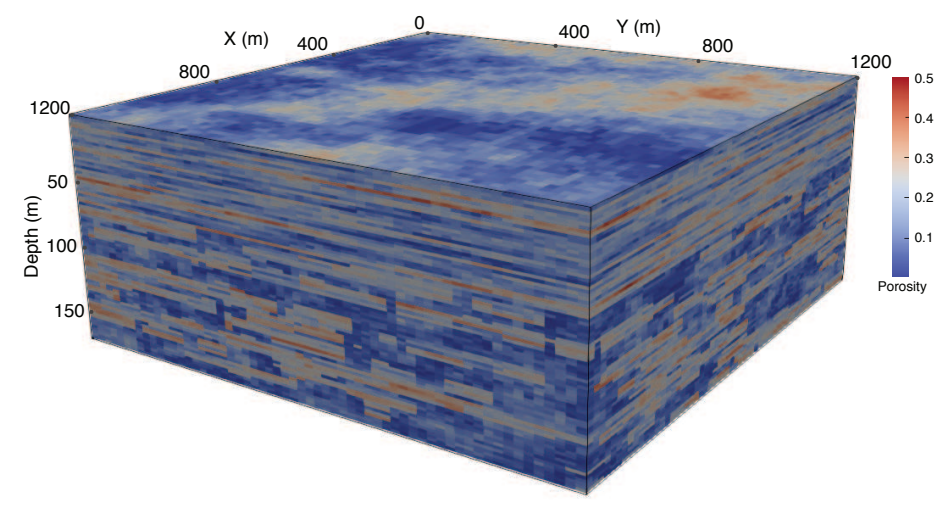

Figure 2: A part of SPE-10 porosity model.

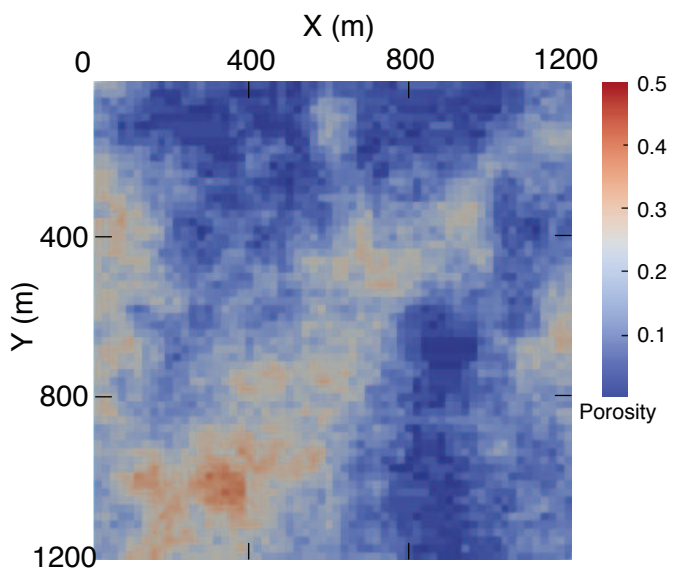

(a)

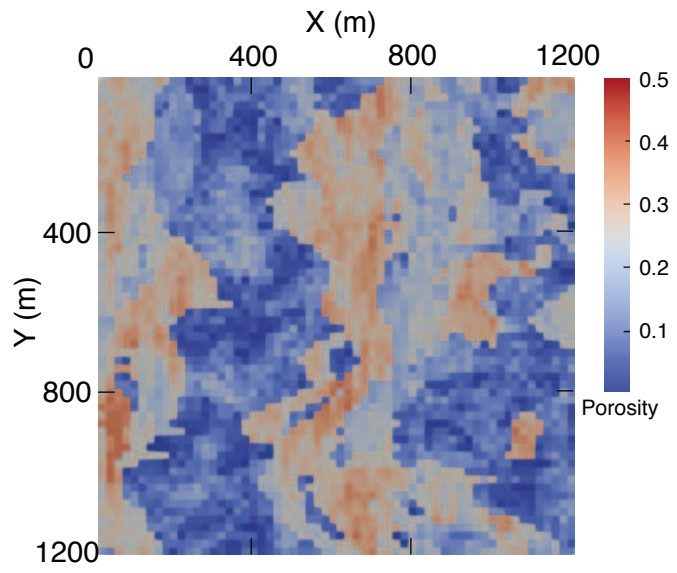

(b)

Figure 3: Comparison of SPE-10 formations in map view: part of (a) Tarbert formation at $50 \mathrm{~m}$ and (b) Upper Ness formation at $150 \mathrm{~m}$ depth.

Tarbert and Upper Ness formation, respectively. The upper formation shows a prograding (coastline advance toward the ocean as a result of the sediment accumulation) near shore environment (Fig. 3(a)), while lower formation is fluvial which includes multiple channels as shown in Fig. 3(b). Note that the region covered by fluvial channels show higher porosity with good connectivity. The SPE-10 model has sufficiently fine layer along the vertical axis, and has simple geological structure with plane top and bottom horizons with no structural variations such as faults. The reason for choosing this model for testing the GMsFEM wave modeling algorithm is that the SPE-10 model has fine vertical layers (20\% of the lateral grids) compared to lateral grid size. Therefore, we can demonstrate how the GMsFEM can capture the fine scale model heterogeneity without employing any averaging or upscaling methods. 


\section{Numerical examples}

\subsection{Fluid simulation and velocity model construction}

First, we performed the fluid simulation by using Eclipse black oil simulator. The fine scale grid size is $10 \mathrm{~m}$ in $x$ and $y$ axis, and $2 \mathrm{~m}$ in $z$ axis. There are a couple major initial conditions for the fluid simulation such as pore pressure and fluid saturation. We applied a uniform fluid pressure as an initial condition of the pressure. The pore pressure on the first day is presented in Fig. 4(a). Note that we used the same visual perspective on the $3 \mathrm{D}$ cube with the pressure volumes for the rest of the 3-D property images shown in this paper. As marked in Fig. 4, the injection well is located at the origin of the coordinate system, and the production well is located at the point $(1200 \mathrm{~m}, 1200 \mathrm{~m})$. Total period of simulation is 700 days, and we inject water to enhance the production volume of hydrocarbon. Fig. 4(a) exhibits that the pore pressure is lower around the production well. Also, as the production proceeds, the pore pressure of the reservoir reduces as displayed in Fig. 4(b). At the production well, as the fluid pressure goes down, the effective stress goes up and the rock stiffens (waves go faster).

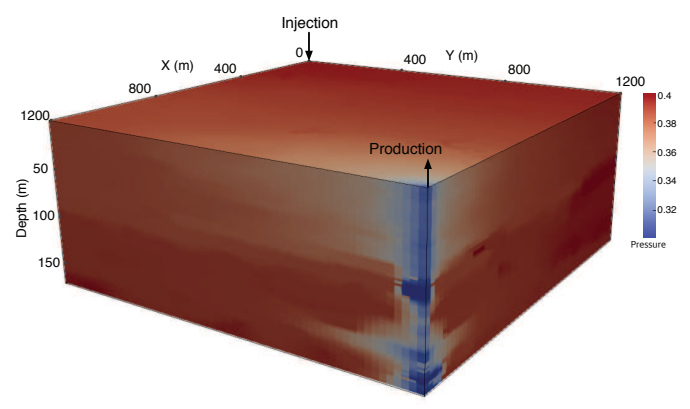

(a)

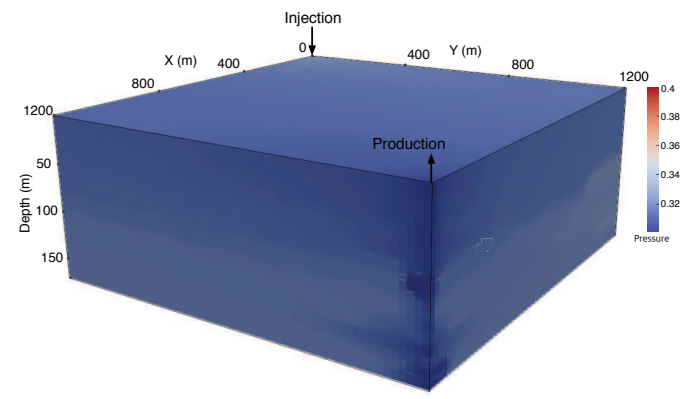

(b)

Figure 4: Pressure $P$ during the injection of water over the production period: pressure at (a) day 1 and (b) day 700 .

Another important initial condition is fluid saturation - the ratio of the fluid composition trapped in pore volumes. Figs. 5(a), 5(c), and 5(e) show the initial fluid saturation. In this case, gas takes the largest volume of the pore, and water and oil share the rest of the volume equivalently. After 700 days of water injection and hydrocarbon production, we could get the fluid saturation as presented in Figs. 5(b), 5(d), and 5(f). Given the fluid saturation volumes after 700 days, we can find that water replaced the rest of the hydrocarbons (gas and oil). In gas saturation, though large portion of gas is produced, there still remains gas around the region with low permeability. One of key goals of this research is to demonstrate whether the GMsFEM wave modeling engine can detect this fine scale fluid variation without performing upscaling. Therefore, after obtaining the outcomes of the fluid simulation, the next step would be generating velocity and density model for the background properties of the wave modeling. 


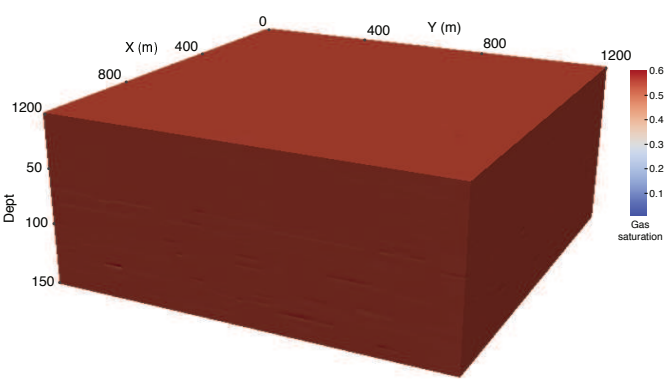

(a)

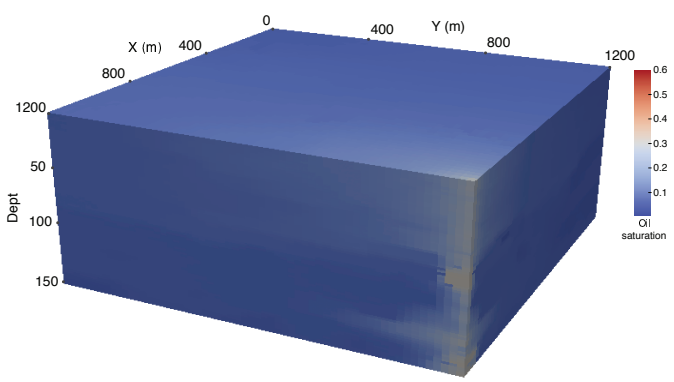

(c)

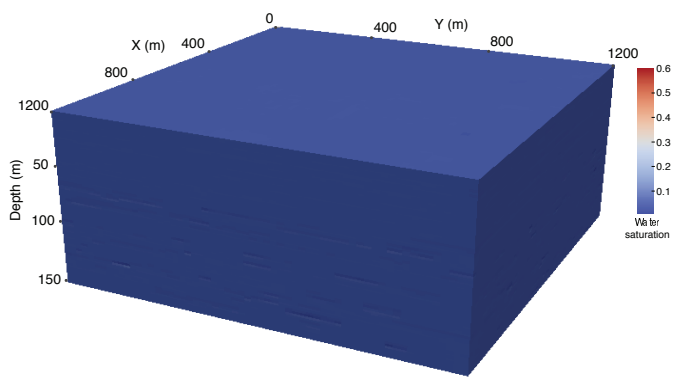

(e)

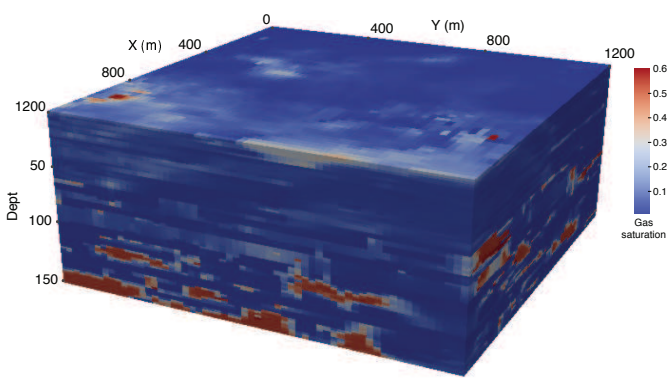

(b)

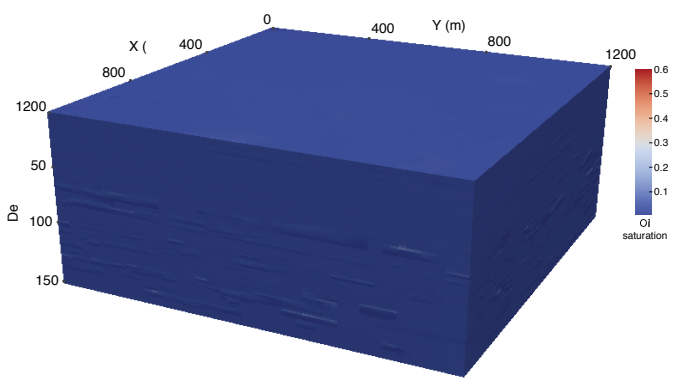

(d)

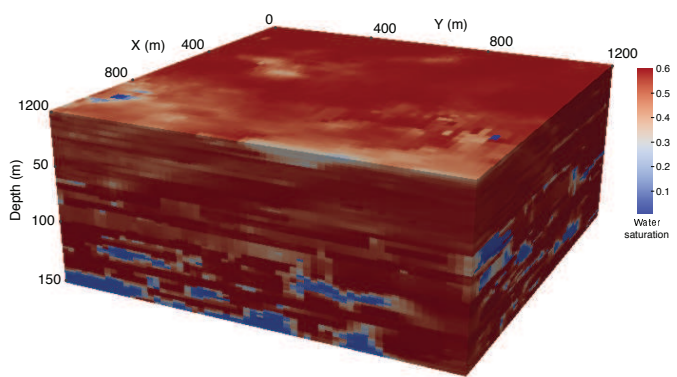

(f)

Figure 5: Comparisons of initial and final fluid saturation over the production period: (a) gas saturation - day 1 , (b) gas saturation - day 700, (c) oil saturation - day 1, (d) oil saturation - day 700, (e) water saturation day 1 , and (f) water saturation - day 700 .

We are able to compute seismic media properties such as density, P- and S-wave velocities using the empirical relation of velocity with reservoir properties [44]. The initial values for the P- and S-wave velocities needed for the Gassmann workflow are calculated with an empirical formula from Eq. (2.2). The distribution of the P-wave velocities in the initial reservoir condition is shown in Fig. 6(a). After generating the velocity volume of the both reservoirs (before and after the production), we subtracted those velocity volumes as $V_{p \text {,Day } 700}-V_{p \text {,Day } 1}$. The volume of P-wave velocity difference is displayed in Fig. 6(b). After a large amount of gas has been produced, the velocity increased since the pore volume is occupied with water instead of gas $\left(v_{w}>v_{g}\right)$. Therefore, the sign of the 


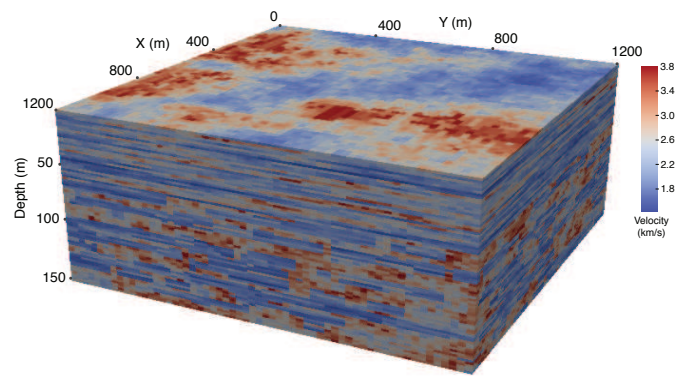

(a)

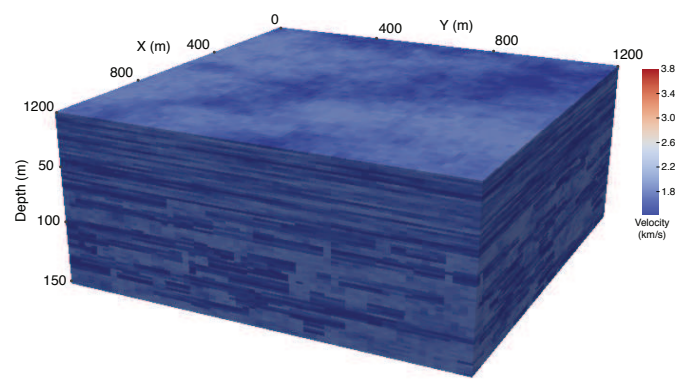

(c)

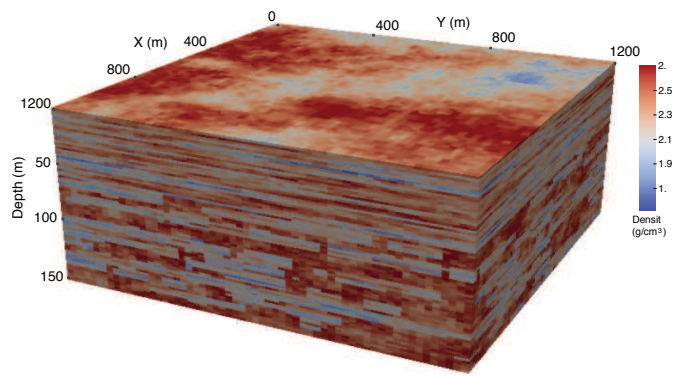

(e)

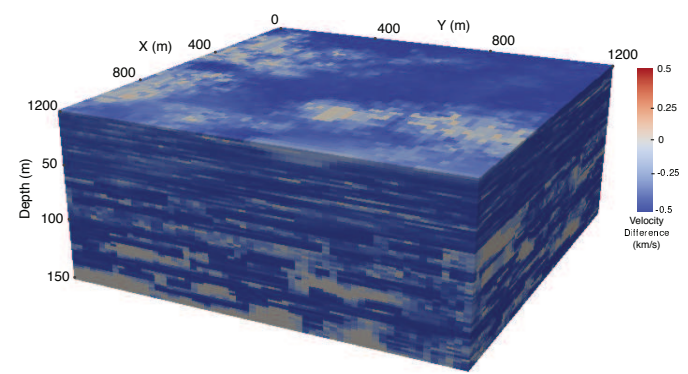

(b)

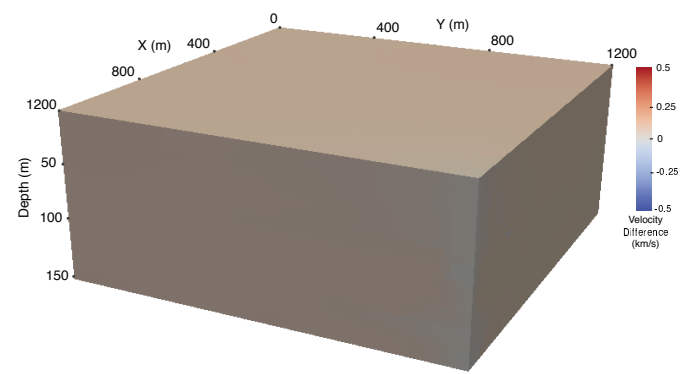

(d)

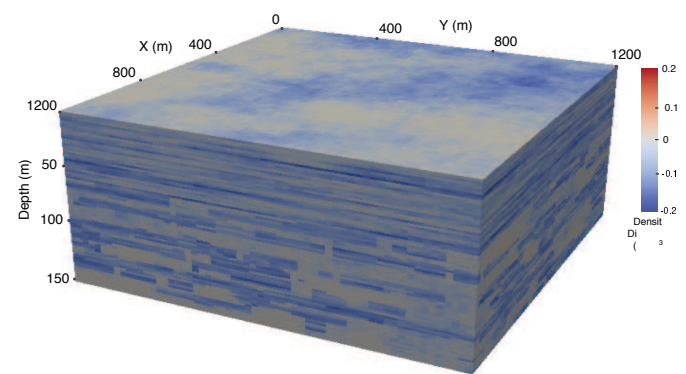

(f)

Figure 6: Calculated initial properties with the difference (Day 700-Day 1): (a) initial P-wave velocity $\left(V_{p, \text { Day } 1}\right)$, (b) difference of $V_{p}\left(V_{p, \text { Day700 }}-V_{p, \text { Day } 1}\right)$, (c) initial S-wave velocity $\left(V_{s, \text { Day } 1}\right)$, (d) difference of $V_{s, \text { Day } 700}-V_{s}\left(V_{s, \text { Day } 1}\right),(\mathrm{e})$ initial density $\left(\rho_{\text {Day } 1}\right)$, and $(\mathrm{f})$ difference of $\rho\left(\rho_{\text {Day } 700}-\rho_{\text {Day } 1}\right)$.

difference volume is positive. In contrast, given the difference volume shown in Fig. 6(d), S-wave velocity shows little change despite the altered fluid composition. A pure change in fluid composition (i.e. same effective stress) will only affect $V_{s}$ through a change in the bulk density. The trend of S-wave velocity follows the trend of the effective pressure (Eq. (2.2)) rather than that of the fluid variation. This phenomena becomes more obvious when we express the P- and S-wave velocity as a function of Lamé constant s. When we assume the elastic properties of isotropic linear case, the wave velocities can be determined as: $V_{p}=\sqrt{(\lambda+2 \mu) / \rho}$ and $V_{s}=\sqrt{\mu / \rho}$, where $\lambda$ is the first Lamé parameter and $\mu$ 
is shear modulus. According to the relations, S-wave is governed by the shear modulus and density, and the shear modulus of the fluid is negligible compared to that of the rock matrix. Therefore, the fluid variation has little influence on the S-wave velocity volume as displayed in Fig. 6(d), and the only effect is due to a change in bulk density. Note that the scale of S-wave difference is less than $25 \mathrm{~m} / \mathrm{s}$, which might be hard to be detected by applying a classical seismic measurement; however, the maximum difference of the P-wave velocity is around $500 \mathrm{~m} / \mathrm{s}$. Figs. 6(e) and 6(f) present the initial density and the differences in the properties, respectively. Considering the sign of the density difference, which is positive, we could assume that the fluid density in pore volume would increase as time goes. In other words, as the water replaces the gas, the bulk density would increase.

Given the differences of the P-wave velocity and density volumes, we would expect that the massive gas production induces the change of the travel time and the amplitude in the time-lapse seismic. In the following section, we are to demonstrate the results of the GMsFEM wave modeling by using these density and velocities as the properties of the elastic media.

\subsection{Multiscale wave modeling}

After extracting the velocity and density information from the fluid simulation volume, we added over- and under-burden layer to the reservoir model. We assumed that the change of seismic properties is only triggered by the change of fluid composition. Also, another hypothesis that we have set in this study is that there is no fluid change on the over- and under-burden layer. Hence, we extended the model in such a way that there is a homogeneous layer above and below the reservoir (Fig. 7(a)). The properties of the homogeneous over- and under-burden layer are as follows: $V_{p}=2500 \mathrm{~m} / \mathrm{s}, V_{s}=1300 \mathrm{~m} / \mathrm{s}$, and $\rho=2000 \mathrm{~m} / \mathrm{s}$. In the designed model, the thickness of the reservoir is $320 \mathrm{~m}$, and we located the source at the center of the model and at a depth of $50 \mathrm{~m}$. We assumed that a horizontal well is drilled for monitoring purpose $\mathrm{s}$, and the receivers are installed in the well at $650 \mathrm{~m}$ depth. By investigating multiple seismic data which is acquired at different times, we will demonstrate the influence of the change of fluid components on the seismic waves (i.e., travel time or amplitude). The mesh used for the GMsFEM wave modeling is presented in Fig. 7(a). The mesh is finely discretized in the reservoir layer and the near surface area. As the source may be unstable when the size of mesh is too coarse to capture the detailed variation around the source location [34,37,45], we finely designed the mesh at near surface. When we applied a local refinement to the mesh without placing the transition zone, the solution of the GMsFEM might include numerical artifacts such as unnecessary reflections around the place with abrupt change of the size of the coarse grid cells, which hinders accurate observation of the change of the time-lapse seismic. Therefore, to suppress the numerical artifacts, we added a transition zone colored in dark gray as presented in Fig. 7(a).

Before modeling the seismic waves at different time step of flow simulations, we first 


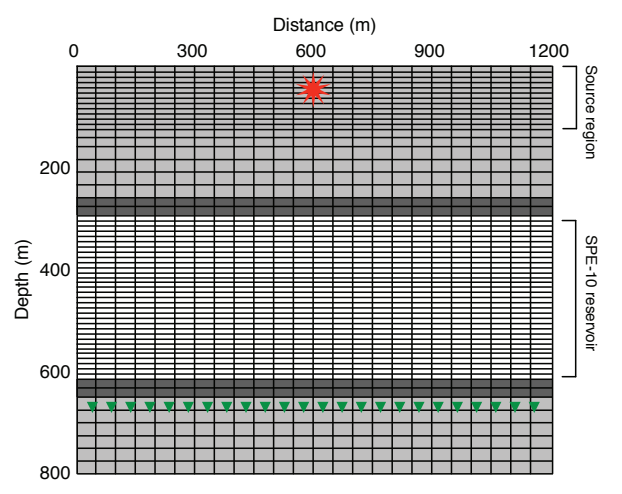

(a)

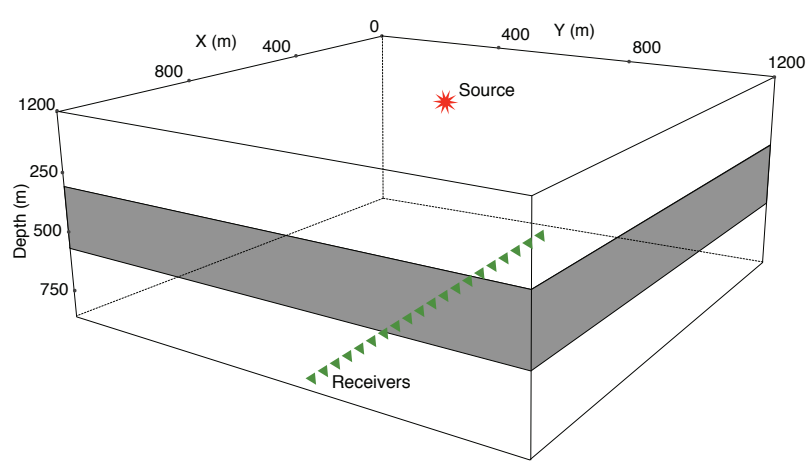

(b)

Figure 7: (a) Coarse scale mesh with (b) acquisition geometry. The red star indicates the seismic source, and the green triangles show output locations (receivers). Variations in mesh cell geometry are described in the text.

demonstrate the accuracy of the GMsFEM solution by comparing the multiscale solutions with the solution of spectral element method (SEM) [13]. For the test, we applied the Ricker wavelet with $20 \mathrm{~Hz}$ central frequency. In SEM, we utilize the fine scale mesh for wave modeling, and consider the solution of SEM as a reference (Fig. 8(a)). The mesh information for the fine scale modeling in the target reservoir is as follows: $d x=10 \mathrm{~m}$, $d y=10 \mathrm{~m}, d z=2 \mathrm{~m}$. We then perform the GMsFEM wave simulation by varying the number of basis functions. We made comparisons among the GMsFEM with different number of basis functions to demonstrate the performance of the proposed method (Table 1). The run-time for computing the basis functions ( $\left.t_{\text {offline }}\right)$ is $1092 \mathrm{~s}$, and the corresponding run-time for the wave modeling $\left(t_{\text {online }}\right)$ is measured by varying the number of basis functions as shown in Table 1. According to the table, increasing the number of interior basis functions more than ten $\left(N_{i}>10\right)$ could not make a tangible difference on the accuracy. Considering the dramatic decrease of the degree of freedom in the GMsFEM, we could expect a large amount of speedup. One might argue that when we include the run-time of the offline stage, we cannot expect an impressive speedup compared to the decreased degree of freedom. However, note that the performance analysis shown in Table 1 is performed by using a wave modeling with a single source. As the multiscale basis functions are independent of the source-receiver geometry, we can apply the same basis functions for simulating the waves with multiple shots. In that case, we can expect a greater amount of speedup by employing the GMsFEM.

Fig. 8 shows the seismic traces which are recorded at the point highlighted with green triangles in Fig. 7(b). As those seismic traces record the displacement as a function of time in different orientations $(x-, y-, z$-directions), we can infer the earth structure by analyzing the seismograms. To put it differently, we can make an indirect measurement of the change of the target reservoir between the source and receivers by observing the difference (Figs. 9 and 10) of the seismic signals at different time steps of the flow simulation.

In the GMsFEM modeling, we applied two different types of multiscale basis func- 
Table 1: Comparison of computing performance of the GMsFEM with different number of interior and boundary basis functions. $t_{\text {online }}$ means the run-time taken for calculating the actual wave simulation though the timemarching. It took $t_{\text {offline }}=1092 \mathrm{~s}$ to compute the multiscale basis functions. Error is measured by calculating the relative $L_{2}$ error.

\begin{tabular}{||cccccc||}
\hline Method & $N_{i}$ & $N_{b}$ & DOFs & $t_{\text {online }}(\mathrm{s})$ & Error \\
\hline SEM & - & - & $17.280 \times 10^{6}$ & 2391.33 & - \\
GMsFEM & 10 & 30 & $1.3824 \times 10^{5}$ & 271.11 & 1.5381 \\
GMsFEM & 10 & 40 & $1.7280 \times 10^{5}$ & 422.42 & 0.4165 \\
GMsFEM & 10 & 50 & $2.0736 \times 10^{5}$ & 566.36 & 0.0118 \\
GMsFEM & 20 & 30 & $1.7280 \times 10^{5}$ & 423.61 & 1.5299 \\
GMsFEM & 20 & 40 & $2.0736 \times 10^{5}$ & 565.87 & 0.4164 \\
GMsFEM & 20 & 50 & $2.4192 \times 10^{5}$ & 699.25 & 0.0092 \\
\hline
\end{tabular}

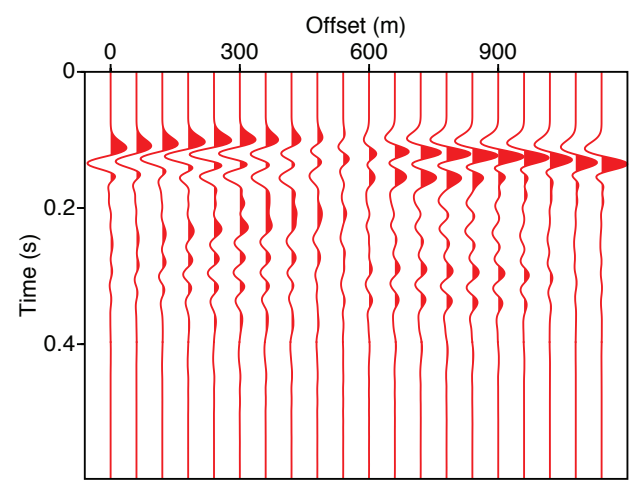

(a)

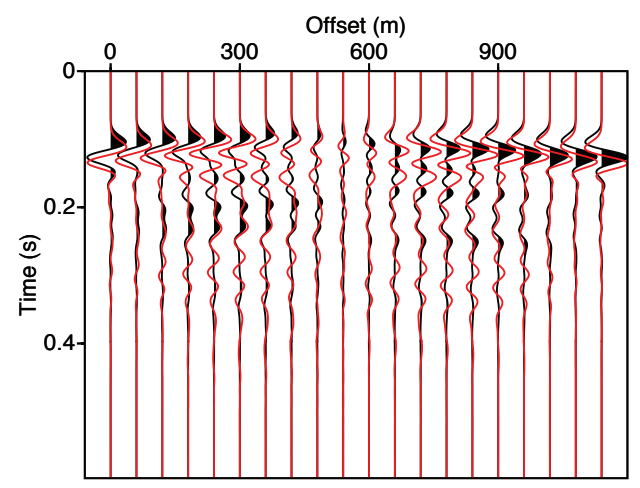

(c)

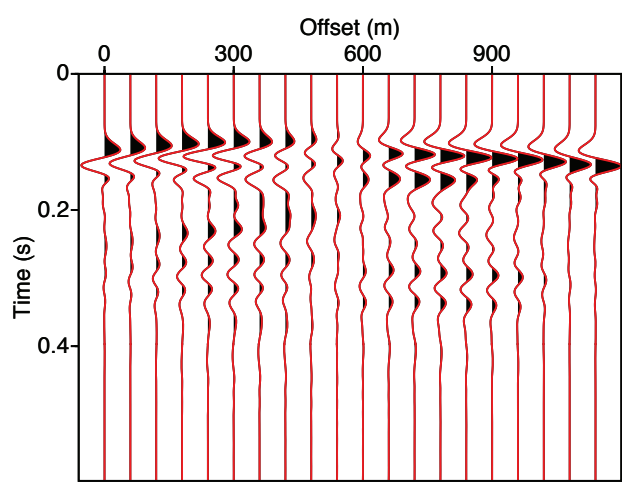

(b)

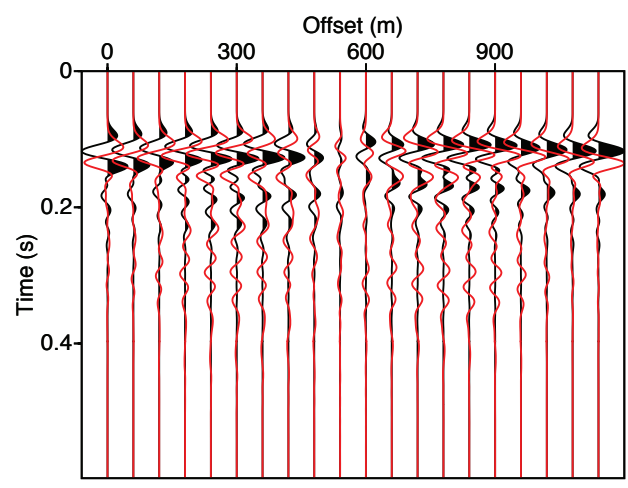

(d)

Figure 8: Comparison of the GMsFEM and SEM $\left(20 \mathrm{~Hz}\right.$ source frequency). Each panel shows $\mathbf{u}_{x}$ component which is calculated from: (a) SEM, (b) GMsFEM $\left(N_{b}=50, N_{i}=10\right)$, (c) GMsFEM $\left(N_{b}=40, N_{i}=10\right)$, and (d) GMsFEM $\left(N_{b}=30, N_{i}=10\right)$. 


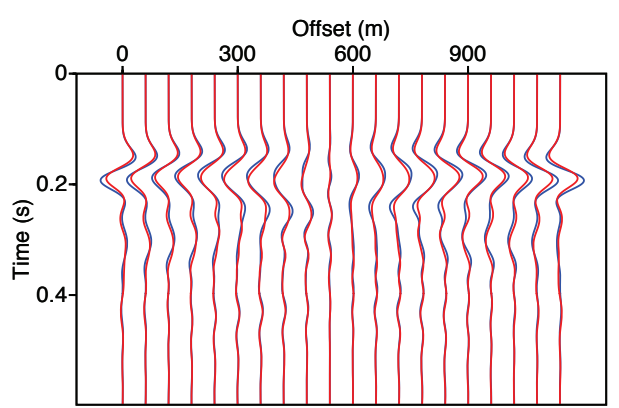

(a) $10 \mathrm{~Hz}, x$-displacement

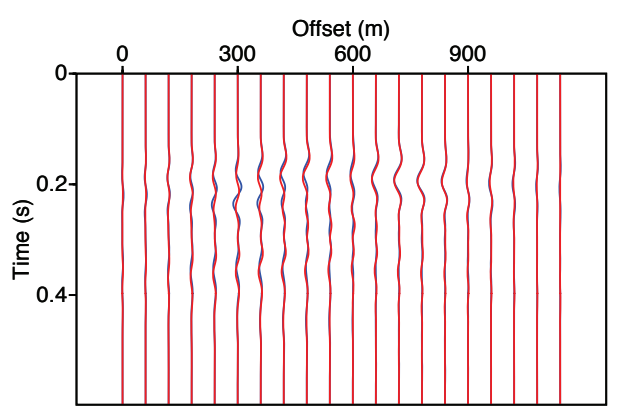

(c) $10 \mathrm{~Hz}, y$-displacement

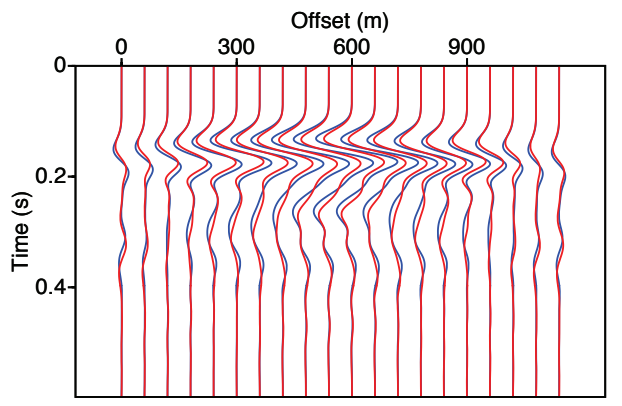

(e) $10 \mathrm{~Hz}, z$-displacement

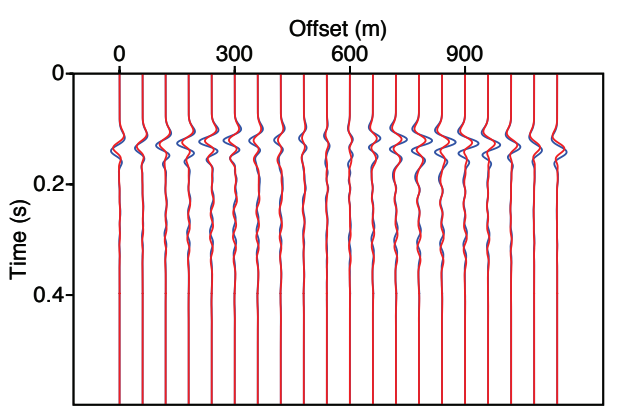

(b) $20 \mathrm{~Hz}, x$-displacement

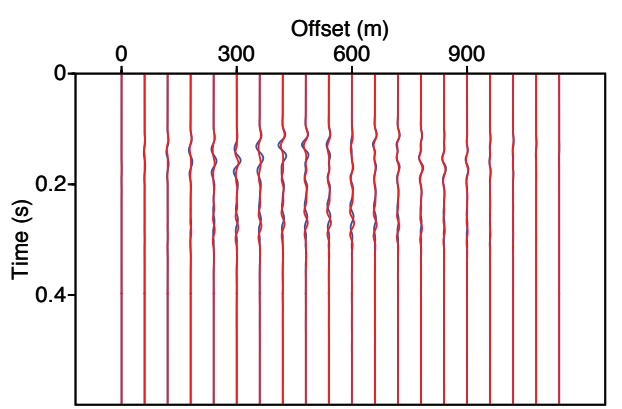

(d) $20 \mathrm{~Hz}, y$-displacement

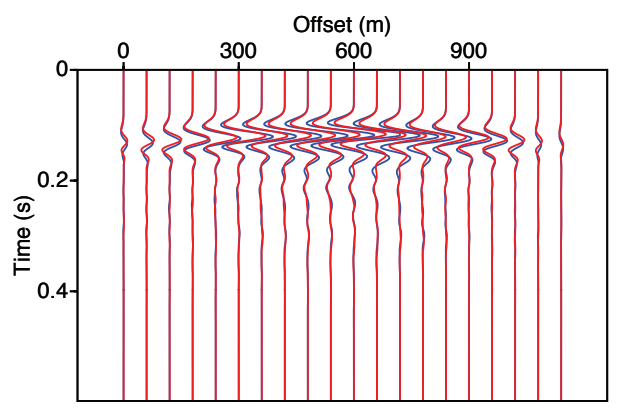

(f) $20 \mathrm{~Hz}$, z-displacement

Figure 9: Time-lapse seismic with different frequencies: $10 \mathrm{~Hz}(\mathrm{a}, \mathrm{c}, \mathrm{e})$ and $20 \mathrm{~Hz}(\mathrm{~b}, \mathrm{~d}, \mathrm{f})$ - blue line (Day 1) and red line (Day 700). Upper, middle, and lower panel present $x-, y-$, and $z$-displacement, respectively.

tions: interior and boundary basis functions. In this case, we fixed the number of interior boundary basis functions to 10, and varied the boundary basis functions from 30 to 50 . The size of coarse mesh is as follows: $d X=50 \mathrm{~m}, d Y=50 \mathrm{~m}, d Z=10 \mathrm{~m}$, so one coarse element includes $125[5 \times 5 \times 5]$ fine elements. We presented the reference solution which is acquired by using the SEM in Fig. 8(a) in red color. Then, we superposed the reference solution to the wave solutions which are obtained from the GMsFEM with various number 


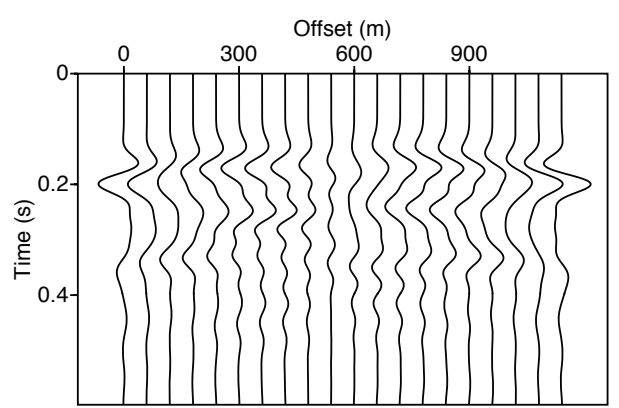

(a)

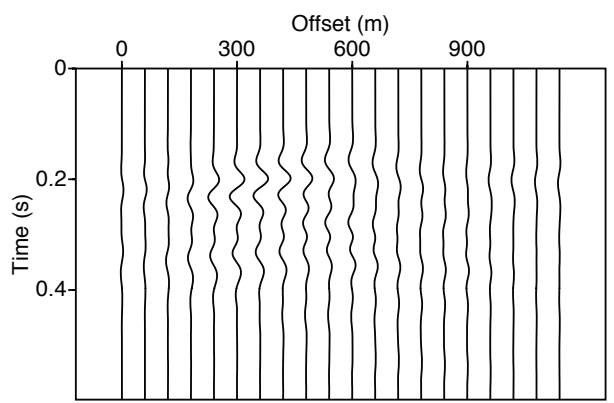

(c)

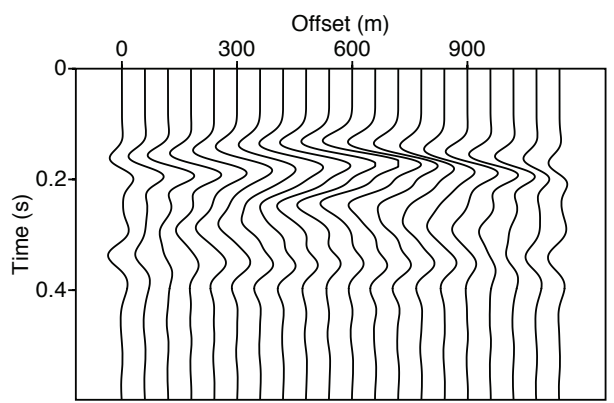

(e)

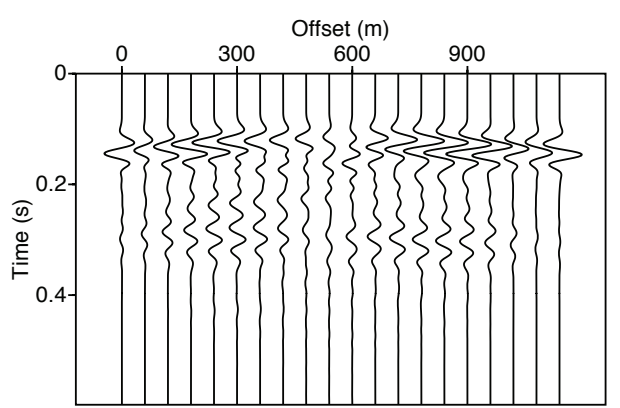

(b)

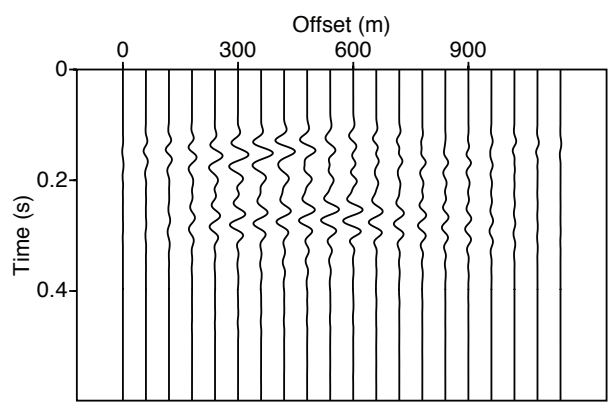

(d)

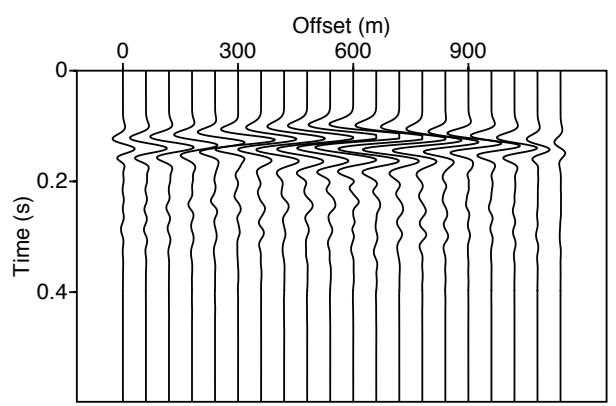

(f)

Figure 10: Time-lapse seismic with different frequencies: $10 \mathrm{~Hz}(\mathrm{a}, \mathrm{c}, \mathrm{e})$ and $20 \mathrm{~Hz}(\mathrm{~b}, \mathrm{~d}, \mathrm{f})$. The upper, middle, and lower panel present the difference of $x-, y-$, and $z$-displacement, respectively.

of basis functions $\left(N_{b}=30,40,50\right.$ and $\left.N_{i}=10\right)$. Observing the run-time displayed in Table 1 and the seismic traces in Fig. 8, there is a trade-off between the computational speed and the accuracy of solutions. In this case, the wave solution (Fig. 8(b)) with 50 basis functions shows good match with the reference solutions, while the other GMsFEM solutions, which use insufficient basis functions, could not compute the detailed variation of the wave solutions. Gao et al. [37] and Chung et al. [21] showed the effect of differ- 
ent combinations of basis functions. They also demonstrated that a smaller number of basis functions may be enough to model the waves with long wavelength. In GMsFEM, as we apply smaller number of basis functions, it provides the solutions more rapidly. However, inappropriate basis functions cannot calculate the correct solution of the high frequency waves. In this case, therefore, we consider 50 boundary and 10 interior basis functions as a good set to obtain the solution with desired level of accuracy in coarse scale simulation. Again, note that while we compute the accurate wave solutions through coarse scale wave modeling, we do not apply any upscaling technique.

Next, we performed the wave simulation using GMsFEM $\left(N_{b}=50, N_{i}=10\right)$ on each property model: before and after injecting water, producing hydrocarbon (mostly gas). In Fig. 9, we compared two different source frequencies $(10 \mathrm{~Hz}$ and $20 \mathrm{~Hz})$ to observe the influence of wavelength to detect fluid changes within the reservoir layers. Left (Figs. 9(a), 9(c), 9(e)) and right (Figs. 9(b), 9(d), 9(f)) panels present the waves from $10 \mathrm{~Hz}$ and $20 \mathrm{~Hz}$ source frequency, respectively. Blue traces are obtained from the wave modeling with initial properties, while red traces are calculated by using the property models after injecting water (or producing gas). For analysis of the seismogram, We first focus on the different displacement components. In both frequencies, we cannot observe tangible differences between before and after the production event in $\mathbf{u}_{y}$. In contrast, $x$ - and $z$-component of the displacement shows obvious changes in time-lapse seismic signals. In the $10 \mathrm{~Hz}$ case, $\mathrm{P}$ and $\mathrm{S}$ waves are in destructive interference due to the long wavelength; however, we can distinguish those signals in $20 \mathrm{~Hz}$ example. To make a clearer comparison, we displayed the difference in two seismic traces (before and after water injection) in Fig. 10. Given the variation of the time-lapse seismic, we can infer the fluid change of the reservoir. For example, seismic wave from the initial model (Day 1) colored in blue line, which pore volume filled with gas, shows larger amplitude (higher impedance contrast) and longer travel time (slower velocity) compared to the seismograms that are computed from the model after two years of production (Day 700) which is displayed in red line in Fig. 9. In other words, the seismic waves (red line) that are generated after injecting water show smaller amplitude variation and earlier arrival time. According to this analysis, the pore volumes in the region, which has smaller amplitude and reduced travel-time, might be filled with the injected water.

\section{Conclusions}

Time-lapse seismic monitoring in a domain with finely layered reservoirs is a computationally intensive procedure due to the high contrast in size of cells discretizing the reservoir and the rest of the domain. In this work, we used permeability and porosity datasets from the SPE-10 model and empirical formulas to build the velocity and density model containing a reservoir. We then applied the GMsFEM with a special coarse grid for the solution of the elastic wave equation in that model and showed that the results are accurate through the comparison with the results produced by the SPEC FEM code. 
Through our test examples, we demonstrated that the GMsFEM can simulate the waves incorporating fine scale heterogeneity without applying model homogenization or upscaling. Observing the time-lapse seismic data, the displacement of $y$-component might include the key information of the region where the fluid substitution occurs. We expect that more information about the spatial distribution of the fluid change can be inferred by extending the seismic survey.

\section{Acknowledgments}

This research has been partially supported by the Crisman and Berg-Hughes Center for Petroleum and Sedimentary Studies in Unconventional Resources project. Also, this research is conducted with the advanced computing resources provided by Texas A\&M High Performance Research Computing. We thanks to Bram Willemsen and anonymous reviewers for providing constructive comments and reviewing the paper. Yalchin Efendiev would like to thank the partial support from NSF 1620318, the U.S. Department of Energy Office of Science, Office of Advanced Scientific Computing Research, Applied Mathematics program under Award Number DE-FG02-13ER26165 and National Priorities Research Program grant NPRP grant 7-1482-1278 from the Qatar National Research Fund. Yalchin Efendiev would also like to acknowledge the support of Mega-grant of the Russian Federation Government (N 14.Y26.31.0013)

\section{References}

[1] A. Malcolm and B. Willemsen, "Rapid 4d fwi using a local wave solver," The Leading Edge, vol. 35, no. 12, pp. 1053-1059, 2016.

[2] R. Alford, K. Kelly, and D. M. Boore, "Accuracy of finite-difference modeling of the acoustic wave equation," Geophysics, vol. 39, no. 6, pp. 834-842, 1974.

[3] Z. Alterman and F. Karal Jr, "Propagation of elastic waves in layered media by finite difference methods," Bulletin of the Seismological Society of America, vol. 58, no. 1, pp. 367-398, 1968.

[4] M. Dablain, "The application of high-order differencing to the scalar wave equation," Geophysics, vol. 51, no. 1, pp. 54-66, 1986.

[5] K. Kelly, R. Ward, S. Treitel, and R. Alford, "Synthetic seismograms: A finite-difference approach," Geophysics, vol. 41, no. 1, pp. 2-27, 1976.

[6] A. R. Levander, "Fourth-order finite-difference p-sv seismograms," Geophysics, vol. 53, no. 11, pp. 1425-1436, 1988.

[7] Y. Liu, "Globally optimal finite-difference schemes based on least squares," Geophysics, vol. 78, no. 4, pp. T113-T132, 2013.

[8] B. A. Bolt and W. D. Smith, "Finite-element computation of seismic anomalies for bodies of arbitrary shape," Geophysics, vol. 41, no. 1, pp. 145-150, 1976.

[9] L. A. Drake and B. A. Bolt, "Finite element modelling of surface wave transmission across regions of subduction," Geophysical Journal International, vol. 98, no. 2, pp. 271-279, 1989.

[10] K. J. Marfurt, "Accuracy of finite-difference and finite-element modeling of the scalar and elastic wave equations," Geophysics, vol. 49, no. 5, pp. 533-549, 1984. 
[11] D. Komatitsch and J.-P. Vilotte, "The spectral element method: an efficient tool to simulate the seismic response of $2 \mathrm{~d}$ and $3 \mathrm{~d}$ geological structures," Bulletin of the seismological society of America, vol. 88, no. 2, pp. 368-392, 1998.

[12] D. Komatitsch, J.-P. Vilotte, R. Vai, J. M. Castillo-Covarrubias, and F. J. Sanchez-Sesma, "The spectral element method for elastic wave equations-application to 2-d and 3-d seismic problems," International Journal for numerical methods in engineering, vol. 45, no. 9, pp. 1139-1164, 1999.

[13] D. Komatitsch and J. Tromp, "Spectral-element simulations of global seismic wave propagationi. validation," Geophysical Journal International, vol. 149, no. 2, pp. 390-412, 2002.

[14] M. F. Wheeler, "An elliptic collocation-finite element method with interior penalties," SIAM Journal on Numerical Analysis, vol. 15, no. 1, pp. 152-161, 1978.

[15] B. Rivière, M. F. Wheeler, and V. Girault, "Improved energy estimates for interior penalty, constrained and discontinuous galerkin methods for elliptic problems. part i," Computational Geosciences, vol. 3, no. 3-4, pp. 337-360, 1999.

[16] D. N. Arnold, F. Brezzi, B. Cockburn, and L. D. Marini, "Unified analysis of discontinuous galerkin methods for elliptic problems," SIAM journal on numerical analysis, vol. 39, no. 5, pp. 1749-1779, 2002.

[17] W. H. Reed and T. Hill, "Triangular mesh methods for the neutron transport equation," tech. rep., Los Alamos Scientific Lab., N. Mex.(USA), 1973.

[18] M. J. Grote, A. Schneebeli, and D. Schötzau, "Discontinuous galerkin finite element method for the wave equation," SIAM Journal on Numerical Analysis, vol. 44, no. 6, pp. 2408-2431, 2006.

[19] M. Dumbser and M. Käser, "An arbitrary high-order discontinuous galerkin method for elastic waves on unstructured meshesii. the three-dimensional isotropic case," Geophysical Journal International, vol. 167, no. 1, pp. 319-336, 2006.

[20] E. T. Chung and B. Engquist, "Optimal discontinuous galerkin methods for wave propagation," SIAM Journal on Numerical Analysis, vol. 44, no. 5, pp. 2131-2158, 2006.

[21] E. T. Chung and B. Engquist, "Optimal discontinuous galerkin methods for the acoustic wave equation in higher dimensions," SIAM Journal on Numerical Analysis, vol. 47, no. 5, pp. 3820-3848, 2009.

[22] T. Bohlen, "Parallel 3-d viscoelastic finite difference seismic modelling," Computers $\mathcal{E}$ Geosciences, vol. 28, no. 8, pp. 887-899, 2002.

[23] D. Komatitsch, D. Michéa, and G. Erlebacher, "Porting a high-order finite-element earthquake modeling application to nvidia graphics cards using cuda," Journal of Parallel and Distributed Computing, vol. 69, no. 5, pp. 451-460, 2009.

[24] T. Y. Hou and X.-H. Wu, "A multiscale finite element method for elliptic problems in composite materials and porous media," Journal of computational physics, vol. 134, no. 1, pp. 169$189,1997$.

[25] Y. Efendiev and T. Y. Hou, Multiscale finite element methods: theory and applications, vol. 4. Springer Science \& Business Media, 2009.

[26] L. Jiang, Y. Efendiev, and V. Ginting, "Analysis of global multiscale finite element methods for wave equations with continuum spatial scales," Applied Numerical Mathematics, vol. 60, no. 8, pp. 862-876, 2010.

[27] E. T. Chung, Y. Efendiev, and R. Gibson, "Multiscale finite element modeling of acoustic wave propagation," in 2011 SEG Annual Meeting, pp. 2898-2903, Society of Exploration Geophysicists, 2011.

[28] E. T. Chung, Y. Efendiev, and R. L. Gibson Jr, "An energy-conserving discontinuous mul- 
tiscale finite element method for the wave equation in heterogeneous media," Advances in Adaptive Data Analysis, vol. 3, no. 01n02, pp. 251-268, 2011.

[29] R. L. Gibson Jr, K. Gao, E. Chung, and Y. Efendiev, "Multiscale modeling of acoustic wave propagation in 2d media," Geophysics, vol. 79, no. 2, pp. T61-T75, 2014.

[30] Y. Efendiev, J. Galvis, and X.-H. Wu, "Multiscale finite element methods for high-contrast problems using local spectral basis functions," Journal of Computational Physics, vol. 230, no. 4, pp. 937-955, 2011.

[31] Y. Efendiev, J. Galvis, and T. Y. Hou, "Generalized multiscale finite element methods (gmsfem)," Journal of Computational Physics, vol. 251, pp. 116-135, 2013.

[32] E. T. Chung, Y. Efendiev, R. L. Gibson Jr, W. T. Leung, et al., "Generalized multiscale finite element modeling of acoustic wave propagation," in 2013 SEG Annual Meeting, Society of Exploration Geophysicists, 2013.

[33] Y. Cho, R. L. Gibson Jr, M. Vasilyeva, and Y. Efendiev, "Generalized multiscale finite elements for simulation of elastic-wave propagation in fractured media," Geophysics, vol. 83, no. 1, pp. WA9-WA20, 2018.

[34] M. Artemyev, H. Baek, R. L. Gibson Jr, et al., "Fast generalized multiscale fem for complex media: Effortless modeling of topography and heterogeneity," in 2015 SEG Annual Meeting, Society of Exploration Geophysicists, 2015.

[35] J. A. Trangenstein and J. B. Bell, "Mathematical structure of the black-oil model for petroleum reservoir simulation," SIAM Journal on Applied Mathematics, vol. 49, no. 3, pp. 749783, 1989.

[36] D. Eberhart-Phillips, "Empirical relationships among seismic velocity, effective pressure, porosity, and clay content in sandstone," Geophysics, vol. 54, pp. 82-, Jan. 1989.

[37] K. Gao, S. Fu, R. L. Gibson, E. T. Chung, and Y. Efendiev, "Generalized multiscale finiteelement method (gmsfem) for elastic wave propagation in heterogeneous, anisotropic media," Journal of Computational Physics, vol. 295, pp. 161-188, 2015.

[38] M. Israeli and S. A. Orszag, "Approximation of radiation boundary conditions," Journal of computational physics, vol. 41, no. 1, pp. 115-135, 1981.

[39] E. T. Chung, Y. Efendiev, and S. Fu, "Generalized multiscale finite element method for elasticity equations," GEM-International Journal on Geomathematics, vol. 5, no. 2, pp. 225-254, 2014.

[40] B. Riviere, Discontinuous Galerkin methods for solving elliptic and parabolic equations: theory and implementation. SIAM, 2008.

[41] M. J. Grote and D. Schötzau, "Optimal error estimates for the fully discrete interior penalty $\mathrm{dg}$ method for the wave equation," Journal of Scientific Computing, vol. 40, no. 1-3, pp. 257272, 2009.

[42] E. T. Chung, Y. Efendiev, and W. T. Leung, "Generalized multiscale finite element methods for wave propagation in heterogeneous media," Multiscale Modeling $\mathcal{E}$ Simulation, vol. 12, no. 4, pp. 1691-1721, 2014.

[43] M. Christie, M. Blunt, et al., "Tenth spe comparative solution project: A comparison of upscaling techniques," in SPE Reservoir Simulation Symposium, Society of Petroleum Engineers, 2001.

[44] T. M. Smith, C. H. Sondergeld, and C. S. Rai, "Gassmann fluid substitutions: A tutorial," Geophysics, vol. 68, no. 2, pp. 430-440, 2003.

[45] S. Fu and K. Gao, "A fast solver for the helmholtz equation based on the generalized multiscale finite-element method," Geophysical Journal International, vol. 211, no. 2, pp. 819-835, 2017. 